\title{
FLAME SPREAD OVER SOLID FUELS IN OPPOSITE NATURAL CONVECTION
}

\author{
E. FERNÁNDEZ-TARRAZO ${ }^{1}$ AND A. LIÑÁN ${ }^{2}$ \\ ${ }^{1}$ Instituto Nacional de Técnica Aeroespacial \\ Madrid, Spain \\ ${ }^{2}$ E.T.S.I. Aeronáuticos \\ $U P M$ \\ Madrid, Spain
}

\begin{abstract}
The downward spread of a flame along the surface of a thermally thick solid fuel inclined at an angle to the vertical is investigated. The flow and the heat transfer in the flame front region that controls the spread rate are described numerically using a single-step Arrhenius model for the gas-phase reaction. The effect of the finite rate kinetics is discussed in terms of a Damköhler number defined as the square of the ratio of the speed of the planar stoichiometric flame of the fuel vapor to the characteristic speed induced by buoyancy in the flame front region. The computed spread rate is lower when the burning surface faces downward than when it faces upward due to the influence of the pressure gradient parallel to the surface.
\end{abstract}

\section{Introduction}

The problem of flame spread over solid fuels has received considerable attention over the years. The classic paper by De Ris [1] dealing with flame spread in the Burke-Schumann limit in opposed forced flow established the basis of theoretical research on the subject. Experimental research conducted by Fernández-Pello and Williams [2] showed basic aspects of the flame structure and the dependence of flame spread velocity on solid surface inclination with respect to the gravity. Based on these results, Fernández-Pello and Williams [3] developed a theory relaxing some of the assumptions made by De Ris. Wichman et al. [4] and Wichman and Williams [5] considered a given uniform shear flow; their results, as those obtained by numerical analysis by Bhattacharje et al. [6], showed the importance of the fluid flow in the determination of the flame propagation velocity.

Some aspects of the structure of the flame are qualitatively well known but difficult to describe either numerically or analytically, such as the triple flame character of the flame front, already recognized by De Ris [1]. The difficulty comes from the high Damköhler numbers required for the triple flame to be identifiable. One way to obtain at least the asymptotic fluid flow and propagation velocity for large Damköhler numbers is to consider the gasphase reaction as infinitely fast. Higuera [7] showed how to treat the singularity that appears when the Burke-Schumann limit is used for the gas-phase reaction, and high activation energy asymptotics, leading to a vaporization temperature, are used to model the solid fuel vaporization. Review papers such as those of Williams [8], Sirignano and Schiller [9], Wichman [10], and Tieszen [11] are available in the literature.

The purpose of this paper is the numerical description of the structure of the leading-edge region of the diffusion flames propagating downward, against the flow due to free convection, along the flat surface of thermally thick solid fuels. We shall account in our analysis for the effects of the inclination $\varphi$ of the solid surface with the vertical; a numerical analysis of these effects for thermally thin solids, and reference to experiments, is given by Chen and Yang [12].

The diffusion flame in the gas phase is maintained by the fuel gasification of the solid as a result of the conductive heating of the solid to the vaporization temperature to initiate the gasification and the reaction in the gas-phase diffusion flame. This flame spreads rapidly upward along the solid, as shown by Tizón et al. [13], and slowly downward.

The extent of the gasification region of the solid under the gravity force grows rapidly to generate, above the flame front region, a reacting gaseous mixing layer, where the effective values of the Grashoff number are large; thus, it can be described using the boundary layer approximation, neglecting longitudinal heat conduction.

However, the boundary layer approximation can not be used in the region at the lower edge of the diffusion flame, where upstream conduction through the gas phase, against the convective flow associated with the buoyancy forces, is an important mechanism for heating the solid to the vaporization temperature, as was recognized early on by De Ris [1]. The rate of downward flame spread is determined 
by the structure of the flow in the flame front region and the temperature distribution in the adjacent heated domain of the solid.

The mathematical problem describing the structure of this flame front region is given in this paper, together with the appropriate boundary conditions, and solved numerically for some representative values of the physicochemical parameters. Although in the analysis we model the gas-phase reaction with an overall Arrhenius reaction, the results are presented in terms of the planar flame velocity of the stoichiometric mixture of the fuel vapor and air, so as to increase the applicability of the numerical analysis.

When the downward flame spread occurs below the solid fuel surface, overpressures on the solid surface due to the buoyancy force normal to the solid generate a pressure gradient that slows down the upward flow created by the buoyancy forces parallel to the solid surface, thus reducing the rate of flame spread. The direction of the pressure gradient component parallel to the surface changes when the flame lies above the solid surface.

\section{Main Scales and Non-Dimensional Formulation of the Problem for the Flame Front Region}

The characteristic values for the size $l_{N}$ and the gas-phase velocity $U_{N}$ in the flame front region are given by the relations, already described by De Ris [1],

$$
U_{N}^{2} / l_{N}=g \text { and } U_{N} l_{N} / v_{0}=1
$$

indicating (1) that the buoyancy forces are responsible for the acceleration of the gas in the region and (2) the requirement that the local Reynolds number is of order unity to allow for the balance of upstream conduction and diffusion. We thus obtain the values

$$
l_{N}=\left(v_{0}^{2} / g\right)^{1 / 3}, U_{N}=\left(v_{0}\right)^{1 / 3}
$$

The characteristic thickness $l_{s}$ of the heated layer in the solid is given by the relation

$$
l_{\mathrm{s}} / l_{N}=k_{\mathrm{s}}\left(T_{\mathrm{v}}-T_{0}\right) / k_{0}\left(T_{\mathrm{e}}-T_{0}\right)=1 / \mathrm{N}
$$

resulting from equating the heat flux from the gas phase to the heat conducted to the solid. Here, $k_{\mathrm{s}}$ is the conductivity of the solid and $k_{0}$ that of the ambient gas of kinematic viscosity $v_{0} ; T_{0}$ is the initial value of the temperature of the solid and gas, $T_{\mathrm{e}}$ is the stoichiometric flame temperature, and $T_{\mathrm{v}}$ is the vaporization temperature, both defined below.

We shall confine the analysis to the cases where $N$ is of order unity or larger. Then, the heat conduction normal to the solid surface is balanced by the convective transport of heat due to the motion of the solid relative to the flame front with the characteristic velocity $U_{\mathrm{Fc}}$. This leads to the relation, derived by De Ris [1],

$$
U_{\mathrm{Fc}}=U_{N} N^{2} \alpha_{\mathrm{s}} / v_{0}=\left(g v_{0}\right)^{1 / 3} N^{2} \alpha_{\mathrm{s}} / v_{0}
$$

in terms of the solid-phase thermal diffusivity $\alpha_{s}$, giving an estimate of the downward flame spread velocity.

The aim of our analysis is to show how finite rate effects, as well as the inclination angle of the gravity force with respect to the wall, influence the ratio $u_{\mathrm{F}}=U_{\mathrm{F}} / U_{\mathrm{Fc}}$ of the flame spread velocity $U_{\mathrm{F}}$ to the De Ris estimate $U_{\mathrm{Fc}}$.

When we use a coordinate system moving with the front, with the spatial coordinates shown in Fig. 2 scaled with $l_{N}$, the velocity components $u$ and $v$ scaled with $U_{N}$, and the pressure variations $p^{\prime}$ from the ambient hydrostatic value scaled with $\rho_{0} U_{N}^{2}$ we obtain the following system of equations:

$$
\frac{\partial \rho u}{\partial x}+\frac{\partial \rho v}{\partial y}=0
$$

$$
\begin{aligned}
\rho u \frac{\partial u}{\partial x}+\rho v \frac{\partial u}{\partial y}= & -\frac{\partial p^{\prime}}{\partial x}+(1-\rho) \cos \varphi \\
& +\frac{\partial \tau_{x x}^{\prime}}{\partial x}+\frac{\partial \tau_{x y}^{\prime}}{\partial y}
\end{aligned}
$$

$$
\begin{array}{r}
\rho u \frac{\partial v}{\partial x}+\rho v \frac{\partial v}{\partial y}=-\frac{\partial p^{\prime}}{\partial y}-(1-\rho) \sin \varphi \\
+\frac{\partial \tau_{x y}^{\prime}}{\partial x}+\frac{\partial \tau_{y y}^{\prime}}{\partial y} \\
\rho u \frac{\partial Y_{\mathrm{F}}}{\partial x}+\rho v \frac{\partial Y_{\mathrm{F}}}{\partial y}=\frac{1}{\operatorname{Pr} L_{\mathrm{F}}} \\
\left(\frac{\partial}{\partial x}\left(\mu \frac{\partial Y_{\mathrm{F}}}{\partial x}\right)+\frac{\partial}{\partial y}\left(\mu \frac{\partial Y_{\mathrm{F}}}{\partial y}\right)\right)-\omega \\
\rho u \frac{\partial Y_{0}}{\partial x}+\rho v \frac{\partial Y_{0}}{\partial y}=\frac{1}{\operatorname{Pr} L_{\mathrm{O}}} \\
\left(\frac{\partial}{\partial x}\left(\mu \frac{\partial Y_{0}}{\partial x}\right)+\frac{\partial}{\partial y}\left(\mu \frac{\partial Y_{0}}{\partial y}\right)\right)-S \omega \\
\rho u \frac{\partial \theta}{\partial x}+\rho v \frac{\partial \theta}{\partial y}=\frac{1}{\operatorname{Pr}}\left(\frac{\partial}{\partial x}\left(\mu \frac{\partial \theta}{\partial x}\right)+\frac{\partial}{\partial y}\left(\mu \frac{\partial \theta}{\partial y}\right)\right) \\
+(1+S) \omega
\end{array}
$$

and the simplified form of the equation of state

$$
\rho(1+\gamma \theta)=1
$$

where we neglect the spatial variations of the pressure and consider that the mean molecular mass is constant. The density is measured with the ambient gas density $\rho_{0}$, and $\theta=\left(T-T_{0}\right) /\left(T_{\mathrm{e}}-T_{0}\right)$ and $\gamma=\left(T_{\mathrm{e}}-T_{0}\right) / T_{0}$. The stoichiometric flame temperature $T_{\mathrm{e}}$ is defined in terms of $Q$, the heat release per unit mass of fuel, by the relation $\left(T_{\mathrm{e}}-T_{0}\right) / T_{0}$ $=\gamma=Q / c_{\mathrm{p}} T_{0}(1+S)$ 

by

The non-dimensional viscous stresses $\tau^{\prime}{ }_{i j}$ are given

$$
\tau_{i j}^{\prime}=\mu\left(\frac{\partial v_{i}}{\partial x_{j}}+\frac{\partial v_{j}}{\partial x_{i}}\right)
$$

in terms of the viscosity measured with its ambient gas value. We use for $\mu$ a power-law approximation $\mu=(1+\gamma \theta)^{n}$. We use the laws of Fourier and Fick for the diffusivities, with constant values $\operatorname{Pr}, L_{\mathrm{F}}$, and $L_{\mathrm{O}}$ for the Prandtl and Lewis numbers.

For the dimensional consumption rate of the fuel per unit volume, we have used an Arrhenius reaction rate

$$
\omega_{\mathrm{F}}=\rho B e^{-E / R T} Y_{\mathrm{F}} Y_{0_{2}}
$$

with an oxygen consumption rate $s \omega_{\mathrm{F}}$ and a heat release $Q \omega_{\mathrm{F}} \cdot Y_{\mathrm{F}}$ and $Y_{\mathrm{O}_{2}}$ are the mass fractions of fuel and oxygen. In the equations; $Y_{0}=Y_{0_{2}} / Y_{0_{2} \mathrm{O}}$ is scaled with the ambient gas value $Y_{0_{2} \mathrm{O}} ; S=s / Y_{0_{2} \mathrm{O}}$ is the air/fuel stoichiometric ratio.

The resulting form of the non-dimensional reaction rate $\omega$ is given by

$$
\omega=D \rho Y_{\mathrm{F}} Y_{0} \exp \left(\frac{\beta(\theta-1)}{1+\frac{\gamma}{1+\gamma}(\theta-1)}\right)
$$

in terms of the non-dimensional activation energy, or Zeldovich number, $\beta=\left(E / R T_{\mathrm{e}}\right)\left(T_{\mathrm{e}}-T_{0}\right) / T_{\mathrm{e}}$, and the Damköhler number, $D=B Y_{0_{2} \mathrm{O}} \mathrm{e}^{-E / R T_{e}} l_{N} / U_{N}$.

The equation giving the temperature distribution in the solid phase, with the longitudinal coordinate $x$ measured with $l_{N}$ and the transverse distance $y(<0)$ to the interface scaled with $l_{\mathrm{s}}$, takes the form:

$$
u_{\mathrm{F}} \frac{\partial \theta}{\partial x}+\hat{R} m_{\mathrm{s}}^{\prime \prime} \frac{\partial \theta}{\partial y}=\frac{1}{N^{2}} \frac{\partial^{2} \theta}{\partial x^{2}}+\frac{\partial^{2} \theta}{\partial y^{2}}
$$

where $u_{\mathrm{F}}$ is the front velocity $U_{\mathrm{F}}$ scaled with $U_{\mathrm{Fc}}$ and $\hat{R} \dot{m}^{\prime \prime}{ }_{s}(x)$ is a non-dimensional form of the regression rate of the solid interface, obtained by dividing the Arrhenius mass rate of gasification $B_{\mathrm{s}} \exp \left(-E_{\mathrm{s}} / R T\right)$ by $\rho_{0} U_{N}$ and multiplying the result by the parameter $\hat{R}=\operatorname{Prc}_{\mathrm{s}}\left(T_{v}-T_{0}\right) / c_{\mathrm{p}}\left(T_{\mathrm{e}}-T_{0}\right)$, where $c_{\mathrm{s}}$ and $c_{\mathrm{p}}$ are the constant specific heats of the solid and gas.

The characteristic value $T_{\mathrm{v}}$ of the vaporization temperature is defined by the relation

$$
B_{\mathrm{s}} \exp \left(-E_{\mathrm{s}} / R T_{\mathrm{v}}\right)=\rho_{0} U_{N}
$$

so that for the typically large values of $E / R T_{\mathrm{v}}$, the strong dependence of $\dot{m}_{\mathrm{s}}^{\prime \prime}$ with $T$ implies that $\dot{m}_{\mathrm{s}}^{\prime \prime}$ can be neglected for $T_{\mathrm{s}}<T_{\mathrm{v}}$ and $T_{\mathrm{s}} \simeq T_{\mathrm{v}}$ when $m_{\mathrm{s}}^{\prime \prime} \neq 0$.

As boundary conditions at the interface $y=0$, which we consider to be nearly planar in the flame front region, the temperature is continuous, and we can write

$$
\begin{aligned}
& y=0^{+}: \quad u=0 ; \rho v=\dot{m}_{\mathrm{s}}^{\prime \prime}(x) ; \\
& \dot{m}_{\mathrm{s}}^{\prime \prime}\left(Y_{\mathrm{F}}-1\right)=\left[\frac{\mu}{P_{\mathrm{r}} L_{\mathrm{F}}} \frac{\partial Y_{\mathrm{F}}}{\partial y}\right]_{0^{+}} \\
& \dot{m}_{\mathrm{s}}^{\prime \prime} Y_{0}=\left[\frac{\mu}{P_{\mathrm{r}} L_{\mathrm{O}}} \frac{\partial Y_{0}}{\partial y}\right]_{0^{+}} \\
& \mu\left[\frac{\partial \theta}{\partial y}\right]_{y=0^{+}}=\left[\lambda \frac{\partial \theta}{\partial y}\right]_{y=0^{-}}+\dot{m}_{\mathrm{s}}^{\prime \prime} l_{\mathrm{v}}
\end{aligned}
$$

where $l_{\mathrm{v}}=L_{\mathrm{v}} \operatorname{Pr} / \gamma c_{\mathrm{p}} T_{0}$ is the non-dimensional form of the heat of vaporization (per unit mass of fuel), and $\lambda=k_{\mathrm{s}} l_{N} / k_{0} l_{\mathrm{s}}=\left(T_{\mathrm{e}}-T_{0}\right) /\left(T_{\mathrm{v}}-T_{0}\right)$.

When analyzing the gas phase, we have neglected the front spread velocity $U_{\mathrm{F}}$ relative to the typical gas phase velocities $U_{\mathrm{N}}$, because $\alpha_{\mathrm{s}} / v_{0} \ll 1$.

In the solid, we must use the boundary condition $\theta \rightarrow 0$ at $y<0, x \rightarrow-\infty$, or at $y \rightarrow-\infty$, far from the front region. The analysis is based on the assumption that the thickness of the solid is large compared with $l_{\mathrm{s}}$. In the gas phase, $y>0$, far from the solid, and upstream, we use the condition that $u-y, v, Y_{F}, Y_{0}-1, p^{\prime}$, and $\theta$ tend to zero for $|\vec{r}| \rightarrow \infty$.

Downstream, the solution of the problem must evolve toward the self-similar solution of the boundary layer form of the conservation equations and boundary conditions.

The Damköhler number $D$ appearing in equation 13 will be written in terms of a reduced Damköhler number $D a=\left(S_{\mathrm{L}} / U_{\mathrm{N}}\right)^{2}$, with a more clear physical meaning, based on the stoichiometric flame speed $S_{\mathrm{L}}$. For $\beta \gg 1$, they satisfy the relation

$$
\begin{aligned}
& 2 D L_{\mathrm{F}} L_{\mathrm{O}} \operatorname{Pr}^{-1} \beta^{-3}(1+\gamma)^{n-1} \\
& \quad S /\left(1+S=D a=\left(S_{\mathrm{L}} / U_{N}\right)^{2}\right.
\end{aligned}
$$

This reduced Damköhler number almost coincides with the one already used by Altenkirch et al. [14] to correlate succesfully the experimental results. When $D a$ is of order unity or larger, in the flame front region we have chemical reaction times of the order of the residence time. If $D a$ is of order unity, then downstream the reaction time will become small compared with the residence time and the reaction will be diffusion controlled.

\section{Asymptotic Behavior}

Downstream, far from the flame front region, we can assume the chemical reaction to be infinitely fast. The reaction occurs, in this case, in a flame surface. We will use the Shvab-Zeldovich variables $Z=$ $\left(S Y_{\mathrm{F}}-Y_{0}+1\right) /(1+S)$ and $H=Y_{\mathrm{F}}+Y_{0}+$ $\theta-1$, obtained by linear combination of equations 7-9, plus the relation $Y_{\mathrm{F}} Y_{0}=0$ obtained in the limit $D a \rightarrow \infty$. 
The self-similar solution of the problem in the mixing layer downstream of the flame front, as was obtained by Kosdon et al. [15] and by Kim et al. [16], is described next. The self-similar solution can be written in terms of the similarity variable $\eta=$ $(\cos \varphi)^{1 / 4} y / x^{1 / 4}$ in the form: $\psi=x^{3 / 4}(\cos \varphi)^{1 / 4} F(\eta)$, $Z=Z(\eta), H=H(\eta)$, and the vaporization rate $\dot{m}_{\mathrm{s}}^{\prime \prime}=A(\cos \varphi)^{1 / 4} x^{-1 / 4}$ where the stream function is defined such that $\rho u=\psi_{y}$ and $\rho v=-\psi_{x}$. When these expressions are substituted in equations 4-9, the following system of ordinary differential equations is obtained:

$$
\begin{gathered}
\frac{1}{2} \rho U^{2}-\frac{3}{4} F \frac{d U}{d \eta}=(1-\rho)+\frac{d}{d \eta}\left(\mu \frac{d U}{d \eta}\right) \\
F(\eta)=-\frac{4}{3} A+\int_{0}^{\eta} \rho(s) U(s) d s \\
-\frac{3}{4} F \frac{d Z}{d \eta}=\frac{1}{\operatorname{Pr}} \frac{d}{d \eta}\left(\mu \frac{d Z}{d \eta}\right) \\
-\frac{3}{4} F \frac{d H}{d \eta}=\frac{1}{\operatorname{Pr}} \frac{d}{d \eta}\left(\mu \frac{d H}{d \eta}\right)
\end{gathered}
$$

to be solved with the following boundary conditions: at $\eta \rightarrow \infty$ :

$$
U=Z=H=0
$$

at $\eta=0$ :

$$
\begin{aligned}
U(0)= & H(0)-\left(Y_{\mathrm{F}}(0)+Y_{0}(0)\right. \\
& \left.+\theta_{\mathrm{v}}-1\right)=0 \\
A(Z(0)-1) & -\frac{\mu}{P_{\mathrm{r}}} \frac{d Z}{d \eta}(0)=A\left(\lambda \hat{R} \theta_{\mathrm{v}}+l_{\mathrm{v}}\right) \\
& -\mu \frac{d \theta}{d \eta}(0)=0
\end{aligned}
$$

In the above problem, the constant $A$ has to be obtained as part of the solution, which also provides the negative value of $F(\infty)$, determining the air entrainment rate.

The boundary layer description gives an outer form, for $\eta \gg 1$, of the stream function $\psi \sim x^{3 / 4}(\cos$ $\varphi)^{1 / 4} F(\infty)$, which leads to an outer irrotational entrainment flow of the form

$$
\psi=\sqrt{2}(\cos \varphi)^{1 / 4} F(\infty) r^{3 / 4} \sin \frac{3}{4}(\pi-\phi)
$$

This external flow, although giving a zero value for the stream function on the solid surface, does not satisfy the non-slip boundary condition. Thus, we encounter another boundary layer below the flame edge, adjacent to the solid surface, of thickness of order $(-x)^{5 / 8}$, which we need to describe to obtain a reliable representation of the far field flow to be used for an accurate numerical solution of the flame front problem.

\section{Results of the Numerical Analysis}

The mathematical problem given by the system of conservation equations 4-9 and associated boundary conditions, where the non-dimensional flame spread velocity $u_{\mathrm{F}}$ is an eigenvalue, has to be solved numerically. We have used a numerical scheme based on standard central finite differences for spatial discretization and pseudotransient iteration until the prescribed accuracy is obtained.

Due to the slow decay of the flow velocities associated with the entrainment by the reacting boundary layer, the conditions at the boundary of the computational box are based on a composite expansion using the far field perturbations described above.

The mesh was adapted to the solution in such a way that a fine uniform grid is obtained in the flame front region. For the eigenvalue calculation, the algorithm described in Kurdyumov et al. [17] and in Kurdyumov and Fernández-Tarrazo [18] has been used.

The calculations have been carried out for the limiting case of large values, $E_{\mathrm{s}} / R T_{\mathrm{s}} \gg 1$, of the nondimensional activation energy of the gasification reaction, when the only parameter remaining in the formulation is the gasification temperature $T_{\mathrm{v}}$. This is taken as constant, $T_{\mathrm{v}}=663 \mathrm{~K}$, in our calculations, although it is weakly dependent on $U_{\mathrm{N}}$. Even though the formulation is general, our calculations have considered $L_{\mathrm{F}}=L_{\mathrm{O}}=1$ and have been limited to the case of polymethylmethylacrylate burning in air, with $T_{0}=288 \mathrm{~K}$. In this case, $\operatorname{Pr}=0.72, n=0.5, \gamma=$ $S=8, N=0.9, \hat{R}=0.15, \lambda=5$, and $l_{\mathrm{v}}=0.25$.

The gas-phase kinetic parameters are $\beta$, which is also fixed equal to 10 , and the Damköhler number $D a$, which we vary for different values of the inclination angle $\varphi$.

Some of the results of the numerical calculations are summarized in Fig. 1, where the non-dimensional flame spread velocity $U_{\mathrm{F}} / U_{\mathrm{Fc}}$ is represented as a function of the Damköhler number $\left(S_{\mathrm{L}} / U_{\mathrm{N}}\right)^{2}$ for various angles of inclination of the solid surface with respect to the vertical. Here, like Chen and Yang [12], for $U_{N}$ we have used $\left(v_{0} g \cos \varphi\right)^{1 / 3}$, replacing $g$ by its component $g \cos \varphi$ along the surface, because it is the one characterizing the flow in the boundary layer and in the far field.

Notice that the flame spread velocity is slightly smaller for downward flame spread below the solid surface (positive values of $\varphi$ ) than above the surface. This is due to the component of the buoyancy forces normal to the solid surface, which generates a pressure gradient parallel to the solid, counteracting in one case or enhancing in the other the effects of the buoyancy force parallel to the solid. The limiting cases $\varphi=-\pi / 2$ and $\pi / 2$ are illustrative; in the first one, a hot air plume develops behind the flame front, while in the second case, there is no possibility of a hot air plume developing upward and, instead, the 


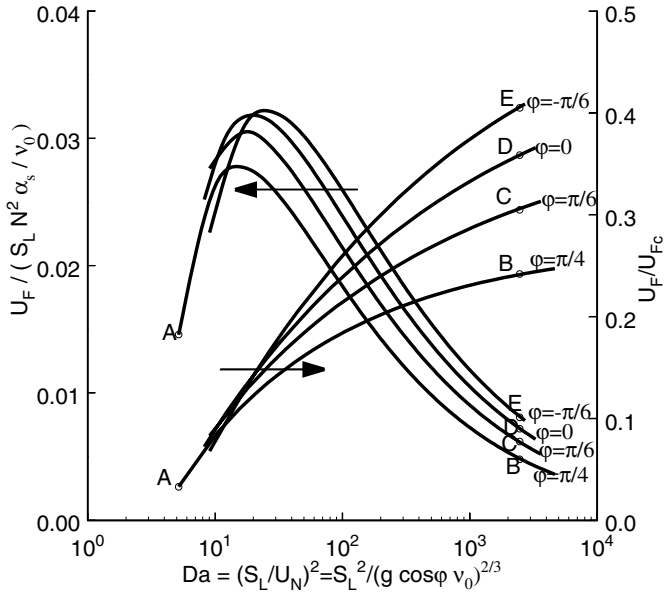

FIG. 1. Non-dimensional flame propagation velocity $U_{\mathrm{F}}$ measured with $U_{\mathrm{Fc}}=\left(g v_{0} \cos \varphi\right)^{1 / 3} N^{2} \alpha / v_{0}$ and non-dimensional flame propagation velocity $U_{\mathrm{F}}$ measured with $S_{\mathrm{L}} N^{2} \alpha_{\mathrm{S}} / v_{0}$ as a function of the Damköhler number for different angles of inclination of gravity.

hot air produced by the diffusion flame moves parallel to the fuel surface in the direction of flame propagation. Experimental evidence was found by Fernández-Pello and Williams [3], showing that when $\varphi$ approaches $\pi / 2$, the flow becomes unstable and the flame propagation is not steady.

The non-dimensional spread rate $u_{\mathrm{F}}$ grows monotonically with $D a$ to the limiting value, of order unity, calculated by Higuera [7] for $D a \rightarrow \infty$. For finite values of $D a$, finite rate effects are important in the flame front region and, thereby, in the determination of $U_{\mathrm{F}} / U_{\mathrm{Fc}}$ because the flame is quenched in a layer close to the surface with a thickness that, when measured with the gravity dependent scale $l_{N}$, increases with decreasing values of the Damköhler number or, equivalently, with increasing values of g, leading, eventually, to flame blowoff.

We show in Fig. 1 the variation of $U_{\mathrm{F}} / S_{\mathrm{L}}$ in terms of the Damköhler number, showing a peak value for a Damköhler number just above blowoff. The peak is due to the decrease of the size of the flame front region as a result of the high velocities induced by gravity and, then, increasing values of the rate of heat transfer.

The form of the streamlines and reaction isocontours in the flame front region are shown in Fig. 2 for cases C, D, and E with a large Damköhler number, indicated in Fig. 1. In these cases, the leading edge of the diffusion flame is a triple flame. The effect of the component of the pressure gradient parallel to the solid in decelerating or accelerating the entrainment flow, for positive or negative values of $\varphi$, is clearly shown in the figure.

Figures 3 and 4 show the variations with $D a$ of the streamlines, reaction isocontours, and isotherms for
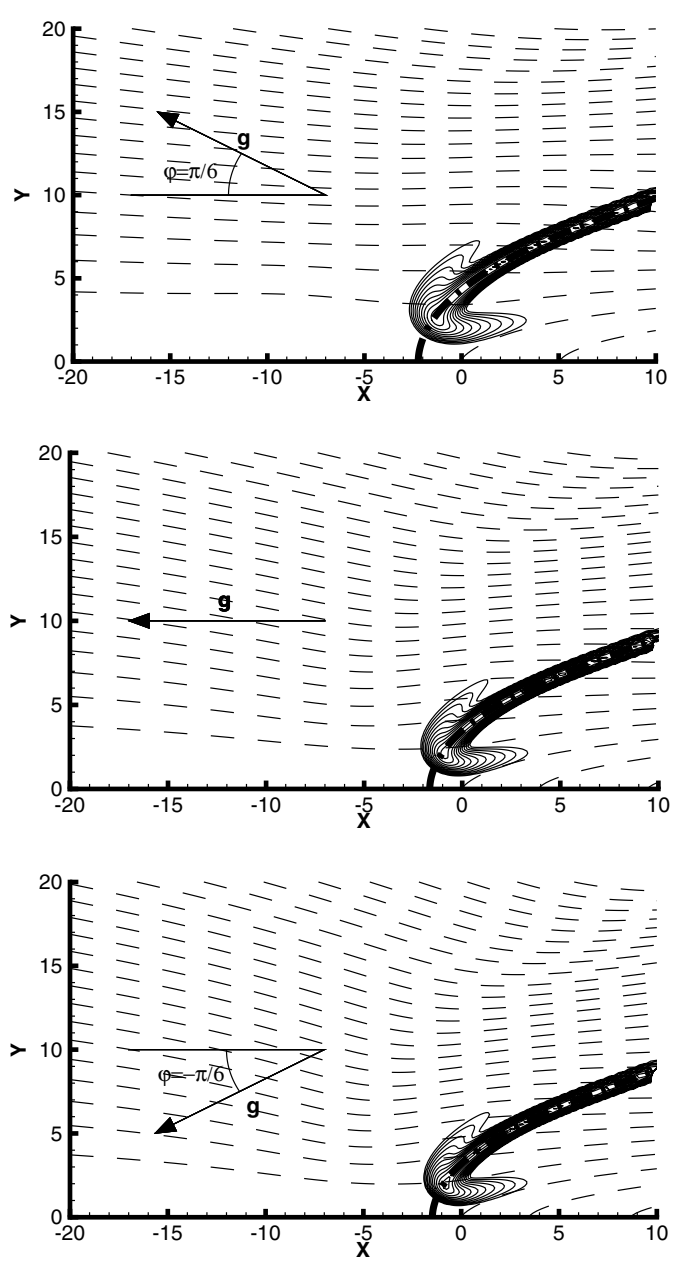

FIG. 2. Flow field for $D a=2.47 \times 10^{3}$. Solid lines: nondimensional reaction rate contours, dashed lines: stream lines, $\psi$ in intervals of 1 , thick dot-dashed lines: stoichiometric line $Z=Z_{\mathrm{s}}$. (top) $\varphi=\pi / 6$, corresponding to point C in Fig. 1 ; (middle) $\varphi=0$, corresponding to point D; (bottom) $\varphi=-\pi / 6$, corresponding to point $\mathrm{E}$.

downstream flame spread with $\varphi=\pi / 4$. Fig. 4 corresponds to case B with a large Damköhler number when the triple flame lies closer to the solid surface. Notice the presence of a recirculating bubble upstream of the flame front, which increases in size with $\varphi$ and $D a$. All these results are in agreement with the experimental results obtained by Fernández-Pello and Williams [2], in particular, the weak dependence on $\varphi$ of the spread rate.

\section{Concluding Remarks}

Although a simplified kinetic mechanism has been used for the description of the flame front structure, 

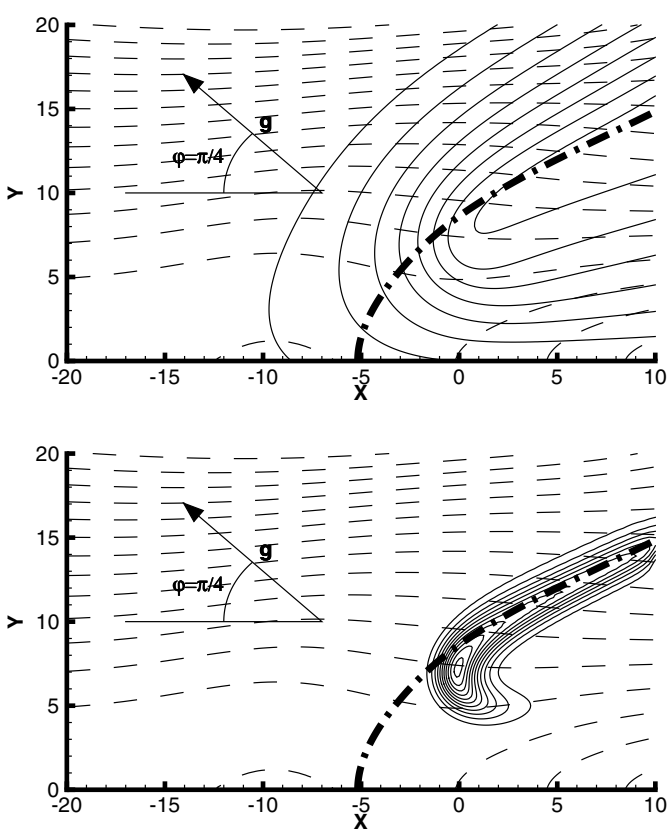

FIG. 3. Flow field for $D a=5.18, \varphi=\pi / 4$, corresponding to point A in Fig. 1. (top) Solid lines: non-dimensional temperature $\theta$ at intervals of 0.1 , dashed lines: stream lines, $\psi$ in intervals of 1 ; thick dot-dashed lines: stoichiometric line $Z=Z_{\mathrm{s}}$. (bottom) Solid lines: non-dimensional reaction rate contours, dashed lines: stream lines, $\psi$ in intervals of 1; thick dot-dashed lines: stoichiometric line $Z=Z_{\mathrm{s}}$.

there are no conceptual difficulties for its generalization to more realistic schemes, and the form of the representation of the results in terms of the stoichiometric flame spread should extend their validity.

When $\varphi$ is increased to values close to $\pi / 2$, the structure of the boundary layer downstream of the flame front region ceases to be dominated by the buoyancy force parallel to the fuel surface. The pressure gradient induced by the normal component of the buoyancy forces pushes the hot gases in the direction of flame spread, so that this is no longer determined by the local structure of the flame front region; there is a change in the flame spreading mechanism. In addition, far from the leading front region, we can expect the flow in the boundary layer to become unstable, although one should not expect large effects of this instability on $U_{\mathrm{F}}$.

\section{Acknowledgments}

This work has been supported by the Instituto Nacional de Técnica Aeroespacial (INTA) under Investigation Program IGB 4400903 and by CICYT under contract number BFM 2001-3691.
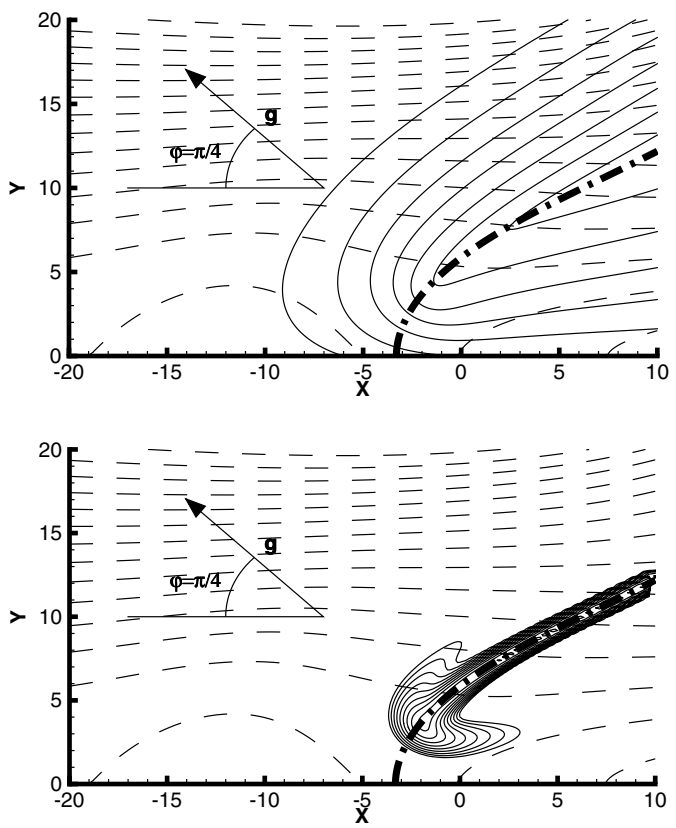

FIG. 4. Flow field for $D a=2.47 \times 10^{3}, \varphi=\pi / 4$, corresponding to point B in Fig. 1. (top) Solid lines: non-dimensional temperature $\theta$ at intervals of 0.1 ; dashed lines: stream lines, $\psi$ in intervals of 1 ; thick dot-dashed lines: stoichiometric line $Z=Z_{\mathrm{s}}$. (bottom) Solid lines: non-dimensional reaction rate contours, dashed lines: stream lines, $\psi$ in intervals of 1 ; thick dot-dashed lines: stoichiometric line $Z=Z_{s}$.

\section{References}

1. De Ris, J. N., Proc. Combust. Inst. 12:241-252 (1968).

2. Fernández-Pello, A., and Williams, F. A., Proc. Combust. Inst. 15:217-231 (1974).

3. Fernández-Pello, A., and Williams, F. A., Combust. Flame 28:251-277 (1977).

4. Wichman, I. S., Williams, F. A., and Glassman, I., Proc. Combust. Inst. 19:835-845 (1982).

5. Wichman, I. S., and Williams, F. A., Combust. Sci. Technol. 32:91-123 (1983).

6. Bhattacharje, S., West, J., and Altenkirch, R., Proc. Combust. Inst. 26:1477-1485 (1996).

7. Higuera, F. J., Combust. Theory Modelling 3:147-158 (1999).

8. Williams, F. A., Proc. Combust. Inst. 16:1281-1294 (1976).

9. Sirignano, W. A., and Schiller, D. N., in Physical and Chemical Aspects of Combustion (F. L. Dryer and R. F. Sawyer, eds.), Gordon and Breach, Amsterdam, 1997, pp. 353-407.

10. Wichman, I. S., Prog. Energy Combust. Sci. 18:553593 (1992).

11. Tieszen, S. R., Annu. Rev. Fluid Mech. 33:67-92 (2001). 
12. Chen, C. H., and Yang, M. T., in Transport Phenomena in Combustion, Vol. 1 (S. H. Chan, ed.), Taylor \& Francis, Washington, DC, 1995, pp. 666-677.

13. Tizón, J. M., Salvá, J. J., and Liñán, A., Combust. Flame 119:41-55 (1999).

14. Altenkirch, R. A., Eichhorn, R., and Shang, P. C., Combust. Flame 37:71-83 (1980).

15. Kosdon, F. J., Williams, F. A., and Buman, C., Proc. Combust. Inst. 12:253-264 (1968).
16. Kim, J. S., De Ris, J., and Kroesser, F. W., Proc. Combust. Inst. 13:949-961 (1970).

17. Kurdyumov, V. N., Fernández-Tarrazo, E., and Liñán, A., Proc. Combust. Inst. 28:1883-1889 (2000).

18. Kurdyumov, V. N., and Fernández-Tarrazo, E., Combust. Flame 128:382-394 (2002).

\section{COMMENTS}

Kuldeep Prasad, NIST, USA. You have employed a single-step mechanism (global) to model the reaction kinetics. Such a model is appropriate for studying diffusion flames. However, for a premixed flame, it is not possible to determine the flammability limit with a global mechanism. These limits are critical for the fuel-rich and fuel-lean branches. Please comment on this.

Author's Reply. For a better description of the leading edge, or triple flame, structure of diffusion flames, we should have used, as you indicate, a reaction model that mimics the variation of the premixed flame propagation velocity with the equivalence ratio. We did not do this in our paper, but it can be achieved by choosing appropriate reaction orders for the fuel and oxygen. However, the contributions of the wings of the premixed flames near the flammability limits to the heat release, which influences the heating of the solid and thereby the flame spread, is small compared to that of the leading edge. The reactants not consumed in the lean and rich wings of the premixed flames will be eventually consumed in the hot layers near the diffusion flame.

James Quintiere, University of Maryland, USA. If this is not discussed sufficiently in the paper, could you amplify on the solution representation at the leading edge as a triple flame? On a similar flame structure for a jet, it was stated that the leading edge flame was not premixed for their extensive kinetics model [1].

\section{REFERENCE}

1. Takahaahi, F., and Katta, V. R., Proc. Combust. Inst. 29:2509 (2002).

Author's Reply. The leading edge structure found in our work shows, when looking at the reaction rate contours, a triple flame for sufficiently large Damköhler numbers or, equivalently, when the velocity of the freely propagating stoichiometric flame is large compared with the typical gas velocity in the flame front region. However, in these cases, the flame front is not propagating freely, and the preheated region upstream of the thin reaction layers reaches the solid surface, and it is not thin compared with the flame standoff distance. With decreasing values of the Damköhler number, the width of the reaction layer of the diffusion flame grows, finally absorbing the reaction layer of the lean premixed flame. Close to extinction, we only see a single reaction layer.

The existence of a partially premixed region close to the solid surface is not limited to Arrhenius kinetic models, although in kinetics that is more realistic the freezing of the reactions in the low temperature region may not affect the recombination reactions. 